\title{
UVES spectra of young brown dwarfs in Cha I: Radial and rotational velocities ${ }^{\star}$
}

\author{
V. Joergens ${ }^{1}$ and E. Guenther ${ }^{2}$ \\ 1 Max-Planck-Institut für Extraterrestrische Physik, Giessenbachstrasse 1, 85748 Garching, Germany \\ 2 Thüringer Landessternwarte Tautenburg, Karl-Schwarzschild-Observatorium, Sternwarte 5, \\ 07778 Tautenburg, Germany
}

Received 3 September 2001 / Accepted 24 September 2001

\begin{abstract}
Based on high-resolution UVES spectra we found that the radial velocity $(R V)$ dispersion of nine of twelve known young bona fide and candidate brown dwarfs in the Cha I dark cloud is $2.0 \mathrm{~km} \mathrm{~s}^{-1}$, i.e. significantly smaller than the $R V$ dispersion of T Tauri stars in Cha I $\left(3.6 \mathrm{~km} \mathrm{~s}^{-1}\right)$ and only slightly larger than the dispersion of the surrounding molecular gas $\left(1.2 \mathrm{~km} \mathrm{~s}^{-1}\right.$ ) (Mizuno et al. 1999). This result indicates that the majority of these brown dwarfs are not ejected with high velocity out of a dense region as proposed by some formation scenarios for brown dwarfs. The mean $R V$ values are consistent with the objects being kinematic members of Cha I. The $R V$ dispersion of the $\mathrm{T}$ Tauri stars confined to the Cha I region is based on a compilation of $\mathrm{T}$ Tauri stars with known $R V \mathrm{~s}$ from the literature plus three $\mathrm{T}$ Tauri stars observed with UVES and unpublished $R V \mathrm{~s}$ for nine $\mathrm{T}$ Tauri stars. Time-resolved spectroscopy revealed $R V$ variations for five out of nine of the bona fide and candidate brown dwarfs in ChaI, which could be due to orbiting planets or surface features. Furthermore we derived rotational velocities $v \sin i$ and the Lithium $6708 \AA$ equivalent width.
\end{abstract}

Key words. stars: low-mass, brown dwarfs - stars: formation - planetary systems: formation - stars: individual: Cha $\mathrm{H} \alpha 1$ to 12, B 34, CHXR 74, Sz 23

\section{Introduction}

It is still an open question how brown dwarfs form. They may form like planets in disks around normal stars. The "brown dwarf desert" is not supporting this theory unless the brown dwarfs are ejected after their formation and can now be detected as freely floating brown dwarfs. Sterzik \& Durison (1999) proposed the ejection of brown dwarfs from cloud cores by three-body encounters.

Brown dwarfs may also form like stars by collapse of a cloud but do not become stars because they formed out of relatively small cores. There are several observational hints to significant circumstellar material around young brown dwarfs (e.g. Comerón et al. 2000 for Cha I) supporting a star-like formation. Recently Reipurth \& Clarke (2001) proposed that brown dwarfs form by cloud fragmentation but failed to become stars because they have been ejected in the early accretion phase.

Understanding the formation of brown dwarfs is important because the number of brown dwarfs is at least equal to the number of stars (Reid et al. 1999). The

Send offprint requests to: V. Joergens,

e-mail: viki@mpe.mpg.de

* Based on observations obtained at the European Southern Observatory at Paranal, Chile in program 65.L-0629 and 65.I-0011. ejection of a (proto-) brown dwarf in the early accretion phase or later might have left observable signs. In this paper we report on high-resolution spectroscopy of young bona fide and candidate brown dwarfs in Cha I, which indicate that there is probably no run-away brown dwarf among them.

The Chamaeleon cloud complex is comprised of three main clouds. Cha I is the most active star forming cloud among them and one of the most promising grounds for observational projects on very low-mass objects. Recently twelve low-mass M6-M8-type objects, Cha $\mathrm{H} \alpha 1$ to 12 , have been detected in the center of ChaI with ages in the range of 1 to 5 Myrs (Comerón et al. 1999, 2000). Their masses are below or near the border line separating brown dwarfs and very low-mass stars. Four of them are confirmed bona fide brown dwarfs $\left(\sim 30\right.$ to $\left.50 M_{\text {Jup }}\right)$ (Neuhäuser \& Comerón 1998, 1999; Comerón et al. 2000).

\section{Spectroscopy}

Using the high-resolution Echelle spectrograph UVES (Dekker et al. 2000) at the VLT, we carried out spectroscopic observations of nine bona fide and candidate brown dwarfs (M6-M8) (Cha Ho 1 to 8 and 12) and three midM-type T Tauri stars (B 34, CHXR 74 and Sz 23) in Cha I between March and May 2000. For each object at least two spectra separated by a few weeks have been obtained. 


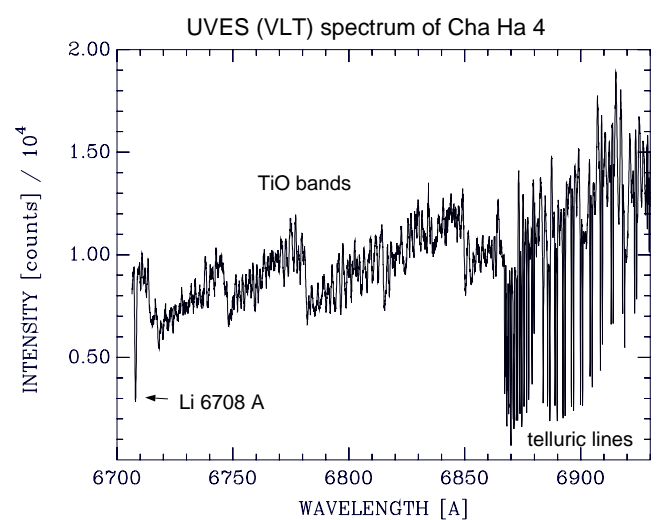

Fig. 1. Small part of an UVES Echelle spectrum of Cha H $\alpha 4$.

UVES is ideal for high precision measurements of $R V \mathrm{~s}$ of faint objects. The spectra have been taken using the red arm of the two-armed UVES spectrograph equipped with a mosaic of two CCDs (blue side of red arm: EEV; red side of red arm: MIT-LL). The wavelength coverage is $6600 \AA$ to $10400 \AA$ and the spectral resolution $\lambda / \Delta \lambda=40000$. The spectra have been taken with a slit of $1^{\prime \prime}$ to $1.2^{\prime \prime}$ during a seeing of $0.4^{\prime \prime}$ to $1.0^{\prime \prime}$.

The spectra have been optimally extracted, including bias correction, flat fielding, cosmic ray elimination and sky subtraction using the Echelle package of $\operatorname{IRAF}^{1}$.

\section{Radial velocities}

For the determination of precise $R V \mathrm{~s}$ we used the telluric $\mathrm{O}_{2}$ lines as wavelength reference. They are produced in the Earth atmosphere and show up in the red part of the optical spectral range (cp. Fig. 1). They are stable up to $\sim 20 \mathrm{~m} \mathrm{~s}^{-1}$ (Balthasar et al. 1982; Caccin et al. 1985).

Heliocentric $R V \mathrm{~s}$ are determined by cross-correlating plenty of stellar lines of the object spectra against a template spectrum and locating the correlation maximum. A mean UVES spectrum of CHXR 74 served as template. The zero point of its velocity has been determined by means of the Lithium line at $6708 \AA$.

For the measurements of Doppler shifts of stellar features we carefully selected appropriate wavelength regions, which are not affected by telluric lines, cosmic defects of the CCD or fringes of the CCD in the near IR. We achieved a $R V$ precision of $\sim 200 \mathrm{~m} \mathrm{~s}^{-1}$ for a $S / N$ of $\sim 20$ in agreement with the expectations for this $S / N$ (Hatzes \& Cochran 1992). The precision of the $R V \mathrm{~s}$ is limited by the $S / N$ of the spectra and not by systematic effects.

The mean heliocentric $R V \mathrm{~s}$ are given in Table 1. They are consistent with $R V \mathrm{~s}$ measured by Neuhäuser \& Comerón (1999) within the measurements uncertainties for Cha $\mathrm{H} \alpha 1,3,4,5,7, \mathrm{~B} 34$ and CHXR 74. However, the values for Cha $\mathrm{H} \alpha 8$ and $\mathrm{Sz} 23$ are discrepant by more than $1 \sigma$ and the $R V$ s for Cha $\mathrm{H} \alpha 2$ and 6 by $2 \sigma$. This may be a hint to long-term single-lined spectroscopic binaries.

${ }^{1}$ IRAF is distributed by the National Optical Astronomy Observatories, which is operated by the Association of Universities for Research in Astronomy, Inc. (AURA) under cooperative agreement with the National Science Foundation.
Table 1. Parameters derived from UVES spectra: mean heliocentric radial velocity $R V$ in $\left[\mathrm{km} \mathrm{s}^{-1}\right]$, projected rotational velocity $v \sin i$ and equivalent width $E W$ of lithium $(6708 \AA)$. Last columns: (sub)stellar radii and upper limits for rotational periods.

\begin{tabular}{lccccc}
\hline object & $\begin{array}{c}R V \\
\pm 0.2\end{array}$ & $\begin{array}{c}v \sin i \\
{\left[\mathrm{~km} \mathrm{~s}^{-1}\right]}\end{array}$ & $\begin{array}{c}E W(\mathrm{Li}) \\
{[\AA]}\end{array}$ & $\begin{array}{c}R_{\star} \\
{\left[R_{\odot}\right]}\end{array}$ & $\begin{array}{c}P_{\max } \\
{[\mathrm{d}]}\end{array}$ \\
\hline $\mathrm{ChaH} \alpha 1$ & 15.5 & $7.6 \pm 2.2$ & $0.14 \pm 0.10$ & 0.46 & 3.1 \\
$\mathrm{ChaH} \alpha 2$ & 15.4 & $12.8 \pm 1.2$ & $0.30 \pm 0.08$ & 0.73 & 2.9 \\
$\mathrm{ChaH} \alpha 3$ & 14.2 & $21.0 \pm 1.6$ & $0.26 \pm 0.08$ & 0.77 & 1.9 \\
$\mathrm{Cha} \mathrm{H} \alpha 4$ & 15.4 & $18.0 \pm 2.3$ & $0.46 \pm 0.11$ & 0.89 & 2.5 \\
$\mathrm{ChaH} \alpha 5$ & 15.5 & $15.4 \pm 1.8$ & $0.44 \pm 0.10$ & 0.83 & 2.7 \\
$\mathrm{ChaH} \alpha 6$ & 16.1 & $13.0 \pm 2.8$ & $0.32 \pm 0.08$ & 0.68 & 2.6 \\
$\mathrm{Cha} \mathrm{H} \alpha 7$ & 13.7 & $\leq 10$ & $\leq 0.09$ & 0.37 & $\geq 1.9$ \\
$\mathrm{ChaH} \alpha 8$ & 14.5 & $15.5 \pm 2.6$ & $0.33 \pm 0.15$ & 0.59 & 1.9 \\
$\mathrm{ChaH} \alpha 12$ & 13.8 & $25.7 \pm 2.6$ & $0.37 \pm 0.10$ & 0.66 & 1.3 \\
\hline B 34 & 16.5 & $15.2 \pm 1.9$ & $0.60 \pm 0.13$ & & \\
$\mathrm{CHXR} 74$ & 15.1 & $14.1 \pm 1.6$ & $0.63 \pm 0.04$ & & \\
Sz 23 & 15.8 & $17.3 \pm 3.4$ & $0.31 \pm 0.08$ & & \\
\hline
\end{tabular}

Due to a low $S / N$ for Cha $\mathrm{H} \alpha 7$ only a mean $R V$ and an upper limit of $v \sin i$ and the Lithium equivalent width could be determined.

\section{Kinematics of brown dwarfs and $T$ Tauri stars in the Cha I cloud}

Neuhäuser \& Comerón (1999) determined a mean $R V$ of $\sim 14.6 \mathrm{~km} \mathrm{~s}^{-1}$ and a total range of $11 \mathrm{~km} \mathrm{~s}^{-1}$ for Cha $\mathrm{H} \alpha 1$ to 8 from medium resolution spectra. The measurements of precise $R V \mathrm{~s}$ for Cha $\mathrm{H} \alpha 1$ to 8 and Cha $\mathrm{H} \alpha 12$ with UVES allow us to study the kinematics of these bona fide and candidate brown dwarfs with high accuracy. We find that their $R V$ s lie close together, only spanning a range of $2.4 \mathrm{~km} \mathrm{~s}^{-1}$. The mean $R V$ is $14.9 \mathrm{~km} \mathrm{~s}^{-1}$ and the $R V$ dispersion is $2.0 \mathrm{~km} \mathrm{~s}^{-1}$ (cp. Table 1 and Fig. 2).

The Cha $\mathrm{H} \alpha$ objects are located at the periphery of one of the six cloud cores (No. 5) in Cha I in a region with a relatively high density of young stellar objects. The mean $R V$ of the molecular gas of the Cha I cloud and also of the cloud core No. 5 is $15.3 \mathrm{~km} \mathrm{~s}^{-1}$ (Mizuno et al. 1999). The mean $R V$ of the studied brown dwarfs is consistent with this velocity of the gas and therefore with the objects being kinematic members of Cha I. Mizuno et al. determined the $R V$ dispersion of the gas of core No. 5 to $1.2 \mathrm{~km} \mathrm{~s}^{-1}$. The brown dwarfs show a slightly larger $R V$ dispersion $\left(2.0 \mathrm{~km} \mathrm{~s}^{-1}\right)$ than the surrounding molecular gas but basically reflect the motion of the gas.

The relatively small $R V$ dispersion of the studied bona fide and candidate brown dwarfs gives suggestive evidence that there is no run-away brown dwarf among them. We cannot rule out that some of them have a larger space velocity dispersion since $R V$ s are tracing only space motions in one dimension. Nevertheless our finding indicates that the majority of the nine $\mathrm{ChaH} \alpha$ objects are not ejected with high velocities out of a dense region as proposed in formation scenarios (Sterzik \& Durisen 1999; Reipurth \& Clarke 2001). Some or all of the brown dwarfs may still have been "ejected" with less than escape velocity into an 


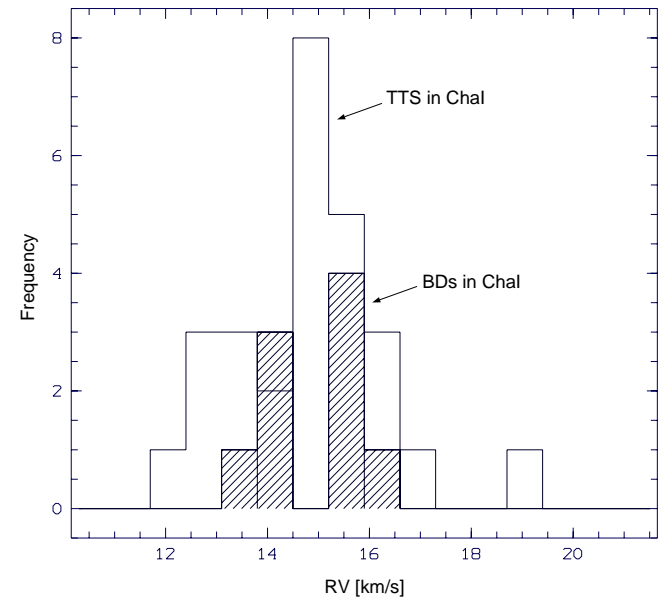

Fig. 2. Histogram of mean $R V$ s of nine bona fide and candidate brown dwarfs (hashed) and for $27 \mathrm{~T}$ Tauri stars in Cha I.

Table 2. List of $\mathrm{T}$ Tauri stars in Cha I with known $R V \mathrm{~s}$ with a precision of $2 \mathrm{~km} \mathrm{~s}^{-1}$ or better from: ${ }^{1}$ Dubath et al. (1996), ${ }^{2}$ Covino et al. (1997), ${ }^{3}$ Guenther et al., in prep. and from UVES observations (B 34, CHXR 74, Sz 23: see Table 1).

\begin{tabular}{|c|c|c|}
\hline object & $R V\left[\mathrm{~km} \mathrm{~s}^{-1}\right]$ & $v \sin i\left[\mathrm{~km} \mathrm{~s}^{-1}\right.$ \\
\hline $\mathrm{Sz} 4^{1}$ & $16.5 \pm 1.3$ & $16.6 \pm 3.3$ \\
\hline $\mathrm{Sz} 6^{1}$ & $14.9 \pm 0.8$ & $38.0 \pm 1.5$ \\
\hline $\mathrm{Sz} 9^{1}$ & $14.7 \pm 0.3$ & $15.2 \pm 1.5$ \\
\hline $\mathrm{Sz} 11^{1}$ & $15.1 \pm 0.5$ & $16.2 \pm 2.3$ \\
\hline $\mathrm{Sz} 15^{1}$ & $17.2 \pm 0.6$ & $4.9 \pm<7.9$ \\
\hline $\mathrm{Sz} 19^{1}$ & $13.5 \pm 0.6$ & $35.7 \pm 1.1$ \\
\hline $\mathrm{Sz} 20^{1}$ & $15.4 \pm 1.3$ & $23.9 \pm 2.7$ \\
\hline $\mathrm{Sz} 36^{1}$ & $12.9 \pm 0.9$ & $7.5(+2.7-4.8)$ \\
\hline $\mathrm{Sz} 41^{1}$ & $13.9 \pm 0.4$ & $34.4 \pm 1.4$ \\
\hline $\mathrm{Sz} 42^{1}$ & $15.1 \pm 0.3$ & $27.2 \pm 1.3$ \\
\hline RX J1109.4-7627 ${ }^{2}$ & $13.1 \pm 2.0$ & $14.5 \pm 3$ \\
\hline $\mathrm{B}_{3}^{2}$ (CHXR 25) & $13.0 \pm 2.0$ & - \\
\hline $\mathrm{F} 34^{2}$ & $14.0 \pm 2.0$ & $55 \pm 3$ \\
\hline RX J1111.7-7620 & $19.0 \pm 2.0$ & $23 \pm 3$ \\
\hline RX J1112.7-7637 2 & $16.0 \pm 2.0$ & $11 \pm 3$ \\
\hline $\mathrm{CS} \mathrm{Cha}{ }^{3}$ & 14.9 & \\
\hline CT Cha ${ }^{3}$ & $15.5 \pm 1.4$ & \\
\hline $\mathrm{CV} \mathrm{Cha}^{3}$ & $15.6 \pm 0.9$ & \\
\hline $\mathrm{SX} \mathrm{Cha}{ }^{3}$ & $13.4 \pm 0.9$ & \\
\hline $\mathrm{SY} \mathrm{Cha}^{3}$ & $12.7 \pm 0.1$ & \\
\hline TW $\mathrm{Cha}^{3}$ & $15.7 \pm 1.2$ & \\
\hline VW $\mathrm{Cha}^{3}$ & $15.1 \pm 0.1$ & \\
\hline VZ $\mathrm{Cha}^{3}$ & 14.7 & \\
\hline WY Cha ${ }^{3}$ & 12.1 & \\
\hline
\end{tabular}

extended orbit around another component of a multiple system. None of the studied brown dwarfs is closer to a known T Tauri star than 4600 AU, i.e. it is unlikely that one of them is still bound to a star. There is still the possibility that the parent star itself was later ejected with escape velocity and left an unbound brown dwarf.

We compared the $R V$ distribution of the bona fide and candidate brown dwarfs also with those of $\mathrm{T}$ Tauri stars. Radio observations by Mizuno et al. (1999) revealed that the three main clouds in the Chamaeleon star forming region differ to a large extent in their star formation properties and also the $R V \mathrm{~s}$ of the molecular gas vary between the clouds (differences up to $3.6 \mathrm{~km}^{-1}$ ), whereas they are relatively constant $\left(\sim 1 \mathrm{~km} \mathrm{~s}^{-1}\right)$ within each single cloud. Therefore it is reasonable to compare the kinematics of the brown dwarfs in Cha I with those of T Tauri stars also confined to the Cha I star forming cloud.

$R V \mathrm{~s}$ of $\mathrm{T}$ Tauri stars in ChaI have been measured by Dubath et al. (1996), Covino et al. (1997), Neuhäuser \& Comerón (1999) and by us and are listed in Table 2. Furthermore unpublished $R V \mathrm{~s}$ of $\mathrm{T}$ Tauri stars based on FEROS spectra have been included (Guenther et al., in prep.). The $\mathrm{T}$ Tauri stars have $R V \mathrm{~s}$ in the range of [12.1. . 19.0 $\left.\mathrm{km} \mathrm{s}^{-1}\right]$ with a mean $R V$ of $14.9 \mathrm{~km} \mathrm{~s}^{-1}$ and a dispersion of $3.6 \mathrm{~km} \mathrm{~s}^{-1}$ (cp. Fig. 2).

The mean $R V$ of the $\mathrm{T}$ Tauri stars matches very well the ones of the bona fide and candidate brown dwarfs in Cha I, whereas the dispersion as well as the total range of $R V$ s of the T Tauri stars is significantly larger than the ones of the brown dwarfs. The stellar activity of T Tauri stars probably account for this discrepancy since it has been shown that $\mathrm{T}$ Tauri stars exhibit a " $R V$ noise" of the order of $\sim 2 \mathrm{~km} \mathrm{~s}^{-1}$ due to stellar activity (Guenther et al. 2000). Moreover it may also play a role that the brown dwarfs and brown dwarf candidates are all situated in a small area at the periphery of one cloud core whereas the T Tauri stars are distributed over the whole Cha I region. The $R V \mathrm{~s}$ of the six cloud cores within Cha I differ by $\pm 0.25 \mathrm{~km} \mathrm{~s}^{-1}$ (Mizuno et al. 1999).

\section{Radial velocity variations}

The analysis of UVES spectra taken at different times yielded to the detection of significant $R V$ variations for five out of nine bona fide and candidate brown dwarfs in ChaI. They could be caused by motion of the brown dwarf due to an orbiting companion or by magnetically induced surface features. The detected $R V$ variations are of the order of $1 \mathrm{~km} \mathrm{~s}^{-1}$. If they are caused by an orbiting object this would have a mass of a few Jupiter masses depending on the orbital parameters (Joergens et al. 2001). More details will be given later after additional follow-up observations.

\section{Projected rotational velocities $v \sin i$}

Projected rotational velocities have been measured using the telluric lines for determining the instrumental profile of the spectrograph and assuming a solar-like center-tolimb variation. We derived $v \sin i$ values in the range from $8 \mathrm{~km} \mathrm{~s}^{-1}$ to $26 \mathrm{~km} \mathrm{~s}^{-1}$ (Table 1 ). Figure 3 shows the $v \sin i$ distribution of the brown dwarfs in comparison with that of T Tauri stars listed in Table 2. Both distributions peak at a $v \sin i$ of 17 to $21 \mathrm{~km} \mathrm{~s}^{-1}$, indicating that there is no crucial difference between the rotational velocities of the studied brown dwarfs and T Tauri stars.

Based on $v \sin i$ and the radius of the object an upper limit of the rotational period can be derived:

$P_{\max }[\mathrm{d}]=50.6145 \frac{R\left[R_{\odot}\right]}{(v \sin i)\left[\mathrm{km} \mathrm{s}^{-1}\right]}$. 


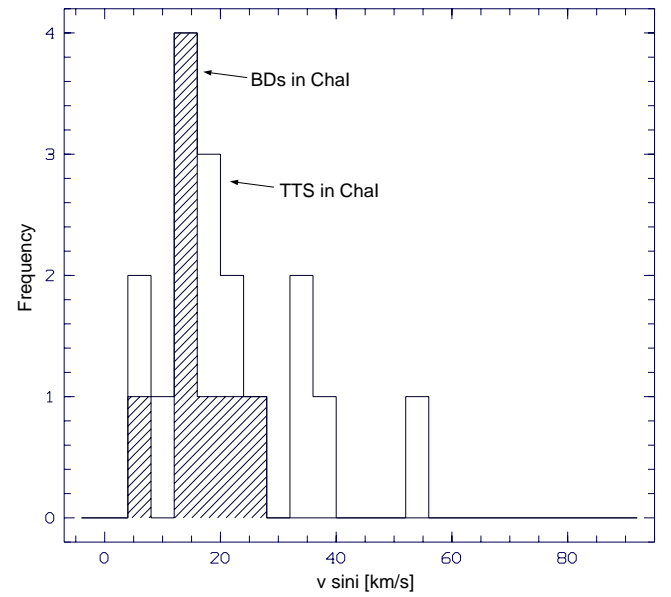

Fig. 3. Histogram of $v \sin i$ for brown dwarfs (hashed) and T Tauri stars in Cha I. For details see Sect. 6.

The radii of the objects are estimated from bolometric luminosities and effective temperatures given by Comerón et al. (2000). The approximate upper limits for the rotational periods are between one and three days (Table 1 ).

\section{Lithium equivalent width}

All brown dwarfs and low-mass stars observed with UVES show lithium absorption at $6708 \AA$. The measured equivalent width is given in Table 1 for all objects. For Cha $\mathrm{H} \alpha 7$ only an upper limit of $E W(\mathrm{Li})$ was determined due to a low $S / N$. For eight of the twelve bona fide and candidate brown dwarfs in Cha I lithium detection and equivalent width measurements have been reported by Neuhäuser \& Comerón (1999). Our UVES spectra allow us to add the Lithium detection of $\mathrm{Cha} \mathrm{H} \alpha 12$.

\section{Summary}

Based on high-resolution UVES spectra of bona fide and candidate brown dwarfs and of $\mathrm{T}$ Tauri stars in Cha I we determined $R V \mathrm{~s}$ with a precision of $\sim 200 \mathrm{~m} \mathrm{~s}^{-1}$.

We found that the $R V$ dispersion of nine of the twelve bona fide and candidate brown dwarfs in Cha I is $2.0 \mathrm{~km} \mathrm{~s}^{-1}$, i.e. significantly smaller than the $R V$ dispersion of T Tauri stars $\left(3.6 \mathrm{~km} \mathrm{~s}^{-1}\right)$ in this cloud and slightly larger than the dispersion of the surrounding molecular gas $\left(1.2 \mathrm{~km} \mathrm{~s}^{-1}\right)$ (Mizuno et al. 1999). This result indicates that the majority of the bona fide and candidate brown dwarfs in Cha I are not ejected with high velocities out of a dense region as proposed in some formation scenarios (Sterzik \& Durisen 1999; Reipurth \& Clarke 2001). Some or all of the brown dwarfs may still have been thrown with less than escape velocity into an extended orbit around another component of a multiple system.

The kinematic study of the $\mathrm{T}$ Tauri stars in Cha I was based on a compilation of all T Tauri stars located in Cha I where $R V \mathrm{~s}$ were known to better than $2 \mathrm{~km} \mathrm{~s}^{-1}$ (including our UVES data for three T Tauri stars as well as unpublished data taken with FEROS). The mean $R V$ of the
T Tauri stars is $14.9 \mathrm{~km} \mathrm{~s}^{-1}$, i.e. the same as for the brown dwarfs. The larger $R V$ dispersion of the $\mathrm{T}$ Tauri stars can at least partly be attributed to " $R V$ noise" caused by stellar activity (Guenther et al. 2000).

Time-resolved spectroscopy revealed significant $R V$ variations for five of the bona fide and candidate brown dwarfs in Cha I, which may be caused by orbiting planets or spots on the surface (Joergens et al. 2001).

Determination of $v \sin i$ showed that the brown dwarfs do not rotate crucially faster than the $\mathrm{T}$ Tauri stars in the same cloud. The $v \sin i$ values together with radii derived from the literature constrained the maximum rotational periods for the individual brown dwarfs to one to three days. Last not least we detected lithium in absorption for all studied objects and measured the equivalent width for Cha $\mathrm{H} \alpha 12$ this is the first lithium detection.

The data presented in this paper showed once more that the brown dwarfs in Cha I form a very homogenous sample and are highly interesting astrophysical objects.

Acknowledgements. We acknowledge helpful discussions on the topic of this paper with R. Neuhäuser, R. Durisen, K. Tachihara and F. Comerón. Furthermore we like to acknowledge the brilliant work of the ESO staff at Paranal. V.J. acknowledges grant from the Deutsche Forschungsgemeinschaft (Schwerpunktprogramm "Physics of star formation").

\section{References}

Balthasar, H., Thiele, U., \& Wöhl, H. 1982, A\&A, 114, 357

Caccin, B., Cavallini, F., Ceppatelli, G., Righini, A., \& Sambuco, A. M. 1985, A\&A, 149, 357

Comerón, F., Rieke, G. H., \& Neuhäuser, R. 1999, A\&A, 343, 477

Comerón, F., Neuhäuser, R., \& Kaas, A. A. 2000, A\&A, 359, 269

Covino, E., Alcalá, J. M., Allain, S., et al. 1997, A\&A, 328, 187

Dekker, H., D'Odorico, S., Kaufer, A., Delabre, B., \& Kotzlowski, H. 2000, in SPIE 4008, ed. M. Iye, A. Moorwood, 534

Dubath, P., Reipurth, B., \& Mayor, M. 1996, A\&A, 308, 107

Guenther, E. W., Joergens, V., Neuhäuser, R., et al. 2000, in Birth and Evolution of Binary Stars, ed. B. Reipurth, \& H. Zinnecker, IAU Symp., 200, in press

Hatzes, A. P., \& Cochran, W. D. 1992, High Resolution Spectroscopy with the VLT, ESO workshop, ed. M. H. Ulrich, 275

Joergens, V., Guenther, E., Neuhäuser, R., et al. 2001, in Origins of stars and planets: The VLT view, ESO workshop, in press [astro-ph/0106185]

Mizuno, A., Hayakawa, T., Tachihara, K., et al. 1999, PASJ, 51,859

Neuhäuser, R., \& Comerón, F. 1998, Science, 282, 83

Neuhäuser, R., \& Comerón, F. 1999, A\&A, 350, 612

Reid, I. N., Kirkpatrick, J. D., Liebert, J., et al. 1999, ApJ, 521,613

Reipurth, B., \& Clarke, C. 2001, ApJ, 122, 432

Sterzik, M. F., \& Durisen, R. H. 1999, in Proc. Star Formation, ed. T. Nakamoto (Nobeyama Radio Obs.), 387 